\title{
Cáncer de Vulva
}

\author{
HOSPITAL UNIVERSITARIO DEL VALLE \\ PERIODO 1974 - 1984
}

\section{Dres.: Jorge Zúñiga C.* y Patricia Cohen**}

En la década de los años 60, recibíamos en el Hospital Universitario del Valle, aproximadamente un caso de Carcinoma de Vulva por año, cuando en contraste, recibíamos en la actualidad casi un caso por mes.

Este hecho tan Ilamativo, sugiere un mayor desarrollo del sistema de referencia de pacientes, el cual como es sabido, se ha implantado con relativo éxito en Cali $y$ en el Valle del Cauca desde hace varios años. Pero es claro también, que ante un incremento en la población afectada por esta entidad, del orden de 12 veces, como anotamos atrás, se hace necesario conocer mejor las características generales y algunas especiales o específicas, si las hubiera, de dicha población.

* Profesor Asociado Univalle. Jefe Servicio de Ginecología. Hospital Universitario Cali.

** Residente 3 Año. Departamento Ginecología y Obstetricia. Hospital Universitario Cali.
Una hipótesis de trabajo sería que esta fuera una enfermedad de la Senilidad, de pacientes de mucha edad; entonces en la misma medida en que aumentara la población a riesgo en nuestro medio se iría aumentando la incidencia de la entidad.

Pero es necesario también tratar de establecer otras características de nuestras pacientes. Si el diagnóstico es demorado no sólo por la responsabilidad de la paciente sino del Médico, cuál es el estado clínico de la enfermedad al momento de la consulta ya que sólo se relaciona con el pronóstico.

Pensamos que las dificultades de diagnóstico son múltiples en nuestro medio.

La idiosincracia del medio sub-desarrollado; la falta de sistemas de salud para hacer un cubrimiento adecuado de la población a riesgo y el pobre nivel educacional.

La falta de recursos para asistir a los centros donde se puede hacer un diagnóstico temprano. 
El hecho de ser una enfermedad de evolución lenta y crónica que afecta la tercera edad tan olvidada y abandonada en nuestro medio.

Se menciona en la literatura una incidencia del 3\% de todos los tumores ginecológicos. Otros hablan del $5 \%$.

La mayoría de las pacientes están entre los 60-70 años. Sólo el $20 \%$ de los casos se presenta antes de los 50 años. En una serie del Hospital M.D. Anderson de la Universidad de Texas (EE.UU.) el $27 \%$ de los casos tenían entre 20 y 50 años.

Green Wifelder y Meiggs han comparado estas pacientes con las de Cáncer Endometrial, son la mayoría de edad avanzada, más en nulíparas, obesas, arterioescleróticas e hipertensas.

La leucoplasia se asocia en el $60 \%$ El 20-30\% de pacientes con estos problemas desarrollan Cáncer de Vulva.

El propósito de este artículo es el de comunicar algunas características de la población atendida por Cáncer de Vulva, en nuestro Hospital en un lapso de 10 años.

\section{PACIENTES $Y$ METODOS}

Se estudiaron 33 pacientes con Diagnóstico de Cáncer de Vulva, atendidas en el Hospital Universitario del Valle entre enero de 1975 y diciembre del año 1984.

En su mayoría eran provenientes del área urbana de la ciudad de Cali, casi todas remitidas de centros de atención básica y diagnosticada la enfermedad por

\begin{tabular}{|l|c|c|}
\hline AREA & CASOS & $\%$ \\
\hline RURAL & 5 & 15.1 \\
\hline URBANA & 28 & 84.9 \\
\hline
\end{tabular}

los médicos que atienden casos de nivel 1 (Centros de Salud Periféricos) de muy precarios recursos económicos y de un estrato social muy bajo.

Para la obtención de los datos, se diseñó el siguiente protocolo.

\section{RESULTADOS}

\section{Procedencia}

En el 63, 64\% (21 casos) procedían del área Urbana de la ciudad de Cali.

En el 30, 30\% (10 casos) eran del resto del Departamento del Valle y sólo un $6.06 \%$ de los casos fueron remitidas de otros sitios. Considerando que muchas de ellas procedían de áreas urbanas de otras ciudades del departamento se agruparon de acuerdo a esa procedencia y se encontró que el $84,84 \%$ ( 28 casos) provenían de esta área.

\begin{tabular}{|l|c|c|}
\hline PROCEDENCIA & CASOS & $\%$ \\
\hline CALI & 21 & 63.6 \\
VALLE & 10 & 30.3 \\
OTROS & 2 & 6.1 \\
\hline
\end{tabular}

Raza

Se clasificó como de raza mestiza el $48.48 \%$ de los casos (16). El 18.18\% (6) casos fueron de raza negra y el $15.15 \%$ (5) casos de raza blanca. En el $18.18 \%$ no estaba en este dato en la historia clínica. 
Este resultado contrasta con varios trabajos que informan la mayoría de los casos en la raza negra por el hecho de padecer más enfermedades granulomatosas de la Vulva.

\section{Edad}

\begin{tabular}{|c|c|}
\hline EDAD & CASOS \\
\hline$<35-$ & 2 \\
$35-44$ & 4 \\
$45-54$ & 6 \\
$55-64$ & 7 \\
$65-74$ & 8 \\
$>75$ & 6 \\
\hline
\end{tabular}

\section{Paridad}

Esta variable es significante en nuestro medio ya que en casi todos los problemas ginecológicos, la mayoría son pacientes grandes multíparas. En este grupo alrededor del $60 \%$ de los casos las pacientes tenían más de 4 hijos.

\begin{tabular}{|c|c|}
\hline PARIDAD & CASOS \\
\hline 0 & 3 \\
\hline 1 & 2 \\
\hline 2 & 5 \\
\hline 3 & 3 \\
\hline 4 & 20 \\
\hline TOTAL & 33 \\
\hline
\end{tabular}

\section{Síntomas}

El síntoma predominante fue el prurito asociado a la presencia de Nódulo en el $30.30 \%$ de los casos. Esto también está presente en la literatura médica; concomitante con la presencia de Ulcera y dolor local.

\begin{tabular}{|l|c|c|}
\hline SINTOMAS & CASOS & $\%$ \\
\hline PRURITO & 10 & 30.3 \\
NODULO & 8 & 24.2 \\
PRURITO Y NODULO & 6 & 18.2 \\
ULCERA Y NODULO & 3 & 9.1 \\
PRURITO Y ULCERA & 2 & 6.1 \\
PRURITO ULCERA & 2 & 6.1 \\
Y NODULO & 1 & 3.0 \\
ULCERA & 1 & 3.0 \\
SIN DATO & 33 & 100.0 \\
TOTAL &
\end{tabular}

Relación con Enfermedades de Transmisión Sexual

Sólo en un porcentaje escaso de las pacientes aparece relación con Enfermedades Sexualmente transmisibles. Es de anotar que en el $40 \%$ de los casos faltó esta pregunta en la historia clínica.

\section{ENFERMEDADES DE TRANSMISION SEXUAL}

\begin{tabular}{|l|c|c|}
\cline { 2 - 3 } \multicolumn{1}{c|}{} & CASOS & $\%$ \\
\hline SIFILIS & 1 & 3.03 \\
\hline GONORREA & 1 & 3.03 \\
\hline CONDILOMA & 1 & 3.03 \\
\hline
\end{tabular}

\section{Sitio de la Lesión}

En el $60 \%$ de los casos la lesión se localizó en los labios mayores o menores. Sólo en $6.1 \%$ en el clirotis ( 2 casos) y un $3.0 \%$ en la Glándula de Bartholin. 


\begin{tabular}{|l|c|c|}
\hline SITIO DE LA LESION & CASOS & $\%$ \\
\hline LABIOS MAYORES & 9 & 27.3 \\
LABIOS MENORES & 9 & 27.3 \\
LABIOS MAYORES & 6 & 18.2 \\
Y MENORES & 4 & 12.1 \\
LABIOS MAYORES & 2 & 6.1 \\
MENORES Y CLITORIS & 1 & 3.0 \\
BARTHOLIS & 1 & 3.0 \\
LABIOS MAYORES & 1 & 3.0 \\
MENORES Y BARTHOLINO & \\
HORQUILLA Y PERINE &
\end{tabular}

Tipo Histológico

\begin{tabular}{|l|c|c|}
\hline HISTOLOGIA & CASOS & $\%$ \\
\hline $\begin{array}{l}\text { CARCINOMA } \\
\text { EPIDERMOIDE }\end{array}$ & 29 & 88 \\
\hline ADENOCARCINOMA & 1 & 3 \\
\hline BASO CELULAR & 1 & 3 \\
\hline SARCOMA & 1 & 3 \\
\hline BOWEN (IN SITU) & 1 & 3 \\
\hline
\end{tabular}

\section{Asociación con otros Tumores}

En un $15.1 \%$ (5 casos) se asoció con Carcinoma de Cervix. En 1 caso 3\% con Cáncer de Vagina; y 1 caso $3 \%$ con Cáncer de Ovario.

OTROS TUMORES ASOCIADOS

\begin{tabular}{|l|c|c|}
\cline { 2 - 3 } \multicolumn{1}{c|}{} & CASOS & $\%$ \\
\hline CANCER DE CERVIX & 5 & 15.15 \\
\hline CANCER DE VAGINA & 1 & 3.03 \\
\hline CANCER DE OVARIO & 1 & 3.03 \\
\hline
\end{tabular}

\section{Estudio de los Ganglios Regionales}

En 15 casos se efectuó vaciamiento inguinal regional. Aproximadamente en el $50 \%$ de estos casos los ganglios se encontraron positivos para metastasis en más de uno.
La ausencia de metastasis a ganglios regionales da una recuperación a 5 años. mayor del $90 \%$; con ganglios positivos. Para metastasis la supervivencia a 5 años es menor del $50 \%$.

La mayoría de casos de este estudio se clasificaron como estadios clínicos II y III, por el tamaño y compromiso de la lesión; con lesiones mayores de $3 \mathrm{cms}$. Hay posibilidad de compromiso ganglionar en el $50 \%$ de los casos.

ESTUDIO DE GANGLIOS REGIONALES (EN 15 VACIAMIENTOS)

\begin{tabular}{|l|c|c|}
\cline { 2 - 3 } \multicolumn{1}{c|}{} & CASOS & $\%$ \\
\hline NEGATIVOS & 8 & 24.2 \\
\hline POSITIVOS & 7 & 21.2 \\
\hline
\end{tabular}

\section{Tratamiento}

La Vulvectomía Radical, se efectuó en 15 caso's $(45.4 \%$ ).

En 10 casos (30.3을 se realizó Vulvectomía Total.

En 1 caso se hizo Hemi-Vulvectomía D. Como tratamiento Paliativo en casos muy avanzados se ordenó Radioterapia en 6 casos.

En ningún caso se utilizó Quimioterapia.

\section{Morbilidad Post-Operatoria}

El principal problema post-operatorio fue la infección Urinaria y la Necrosis de la piel sobre las suturas; problemas de recuperación muy fácil y benigna con manejo médico. 


\begin{tabular}{|c|c|c|}
\hline TRATAMIENTO & CASOS & $\%$ \\
\hline $\begin{array}{l}\text { VULVECTOMIAS } \\
\text { RADICALES }\end{array}$ & 15 & 45.45 \\
\hline $\begin{array}{l}\text { VULVECTOMIAS } \\
\text { TOTALES }\end{array}$ & 10 & 30.30 \\
\hline HEMIVULVECTOMIA & 1 & 3.03 \\
\hline $\begin{array}{l}\text { VULVECTOMIA } \\
+ \text { +RADIOTERAPIA }\end{array}$ & 4 & 12.12 \\
\hline $\begin{array}{l}\text { VULVECTOMIA RADICAL } \\
\text { YRADIOTERAPIA }\end{array}$ & 1 & 3.03 \\
\hline RADIOTERAPIA & 2 & 6.06 \\
\hline
\end{tabular}

No se presentaron casos de Mortalidad post-operatoria inmediata.

MORBILIDAD POST-OPERATORIA

\begin{tabular}{|l|c|c|}
\cline { 2 - 3 } \multicolumn{1}{c|}{} & CASOS & $\%$ \\
\hline $\begin{array}{l}\text { INFECCION } \\
\text { SUTURAS }\end{array}$ & 13 & 39.4 \\
\hline $\begin{array}{l}\text { INFECCION } \\
\text { VIAS URINARIAS }\end{array}$ & 2 & 6.1 \\
\hline
\end{tabular}

\section{Metastasis}

En 9 casos $27.3 \%$ metastasis a la Pelvis.

En 6 casos 18.2\% Metastásis Extra Pélvicas.

\begin{tabular}{|l|c|c|}
\hline METASTASIS & CASOS & $\%$ \\
\hline PELVICAS & 9 & 27.3 \\
\hline EXTRA-PELVICAS & 6 & 18.2 \\
\hline
\end{tabular}

\section{Recidivas}

En 5 casos (15.1\%) se presentó Recidiva Local.

\section{Recuperación a 5 años}

En sólo 8 casos (24.2\%) se tuvo controles a los 5 años, sin evidencia de enfermedad. En el resto de las pacientes se logró un seguimiento entre uno y dos años.
RECIDIVAS TUMORALES

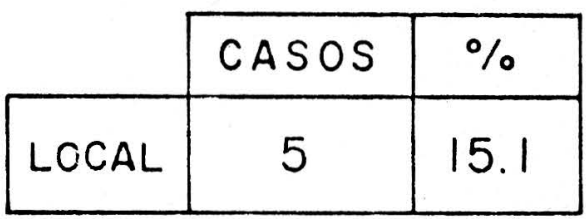

SUPERVIVENCIA (RECUPERACION)

\begin{tabular}{|c|c|c|}
\hline AÑNS & CASOS & \multicolumn{1}{|c|}{$\%$} \\
\hline $1-2$ & 5 & 15.1 \\
$2-3$ & 8 & 24.2 \\
$3-4$ & 3 & 9.1 \\
$4-5$ & 3 & 9.1 \\
5 & 3 & 9.1 \\
6 & 5 & 15.1 \\
\hline SIN DATO & 6 & 18.3 \\
\hline
\end{tabular}

\section{DISCUSION}

El Cáncer Invasivo de la Vulva representa el 3.5\% de los casos de Cáncer de Aparato Genital Femenino. Se considera que la enfermedad predomina en la edad avanzada, la mayoría entre 60-70 años; un $20 \%$ de las pacientes, la edad es menor de 50 años. En la mayoría de los casos el diagnóstico es tardío por negligencia o ignorancia del paciente y aún del médico, no se da importancia al prurito vulvar, y se usa mucho la automedicación antes de hacer una consulta médica formal.

El factor etiológico es confuso, lo más importante son los factores pre. disponentes:

La leucoplasia en el 30 al $50 \%$

Las lesiones granulomatosas están asociadas en el $45 \%$ según informes de Douglas en las Indias Occidentales. 
Las lesiones de origen viral especialmente el Condiloma acuminado, según Charlewood y Shipper 4 de 11 casos de Cáncer de Vulva se relacionan con este problema. Se ha mencionado asociadas la Diabetes y la Arterioesclerosis.

Las lesiones multicéntricas se presentan en el 10 al $20 \%$

En un $10 \%$ de las pacientes con Cáncer de Vulva se presenta Cáncer del Cuello Uterino en alguna etapa de su vida.

Pese a que es una neoplasia que se desarrolla en la parte exterior del cuerpo, las enfermas se presentan con lesiones muy avanzadas, por tener conceptos de pudor exagerados, se practican remedios caseros hasta que el tiempo muestra su inutilidad.

El médico que estudia una lesión en este sitio a menudo lo atribuye a cosas benignas como forúnculos, quistes sebáceos, escoriaciones y dermatitis. Todo esto se da porque son comunes los trastornos alérgicos, la vaginitis atrófica y la leucoplasia.

Clínicamente el Cáncer de Vulva tiene dos características importantes.
A) Es de los pocos tumores malignos dolorosos.

B) La oncología nos enseña que la mayoría de las masas malignas son duras excepto en la Vulva.

Sobre el tipo histólógico en un informe de 1.378 casos publicado en el texto Clinical Gynecologic Oncology se agrupan así:

\section{Epidermoide}

Menaloma

Sarcoma

G. Bartholin

$\begin{aligned} 86.2 & \text { \% } \\ 4.8 & \text { \% } \\ 2.2 & \text { \% } \\ 1.2 & \text { 응 }\end{aligned}$

Según el sitio de localización de la lesión en el texto: "Diseases of the Vulva de N.A. Ja Nouski y C.P. Douglas", se dan los siguientes datos:

Labios mayor y menor

Clítoris

Introito Vaginal

G. Bartholin

Para disminuir el número de errores diagnósticos debe seguirse las siguientes normas que se deben considerar básicas.

1. Toda clase de lesión crónica en la vulva debe ser estudiada con biopsia.

2. No debe utilizarse fulguración ni cauterización sin antes estar seguros de la benignidad o malignidad de la lesión.

3. La leucoplasia debe considerarse como una lesión pre-maligna y necesita vigilarse como tal. Puede estar indicada una Vulvectomía Total.

4. No debe utilizarse radioterapia en el tratamiento de lesiones de la vulva.

\section{CONCLUSIONES}

Presentamos un estudio descriptivo de la Gltima década (1975-1984) del Cáncer de Vulva invasivo en la Clínica de Oncología Ginecológica del Hospital Universitario del Valle.

El $63.63 \%$ procedían de Calicon un $84.84 \%$ de área urbana.

El mayor porcentaje de casos se presenta en el grupo de edad de 65-74 años para un $24.24 \%$ que sumado al $12.12 \%$ de casos por encima de 74 años da un total de $36.36 \%$ por encima de los 65 años. 
La raza mestiza ocupó el porcentaje más al to para un $48.48 \%$

La multiparidad se asoció en un 60 , $60 \%$ (más de 4 partos).

El síntoma predominante fue el prurito asociado a vulvitis en el $30,30 \%$

El tumor asociado más frecuente fue el Carcinoma de Cervix en un $15,15 \%$

El sitio más frecuente de la lesión fueron los labios mayores y menores en un $72,72 \%$

En el 18, $18 \%$ se presentó lesión central (Clítoris).

El Carcinoma Epidermoide fue el más frecuente en el $87,87 \%$

Se encontraron ganglios positivos en el 21, $21 \%$ de los casos.

Metastasis pélvicas y extrapélvicas en el $45,45 \%$ les:

Se practicaron Vulvectomía Radica-

(Vulvectomía total con Vaciamiento Ganglionar Regional) en el 39,39\% de los casos.

Vulvectomías totales en el $30,30 \%$ de los casos. 1 caso.

Hemi-Vulvectomía en el $3,03 \%$,

Radioterapia en el $21,21 \%$ ( 7 casos).

En ningán caso se utilizó Quimioterapia.

La morbilidad Post-Operatoria fue la infección de las suturas en el 39, 39\%.

La supervivencia o recuperación de las pacientes se puede analizar así:

Controles sin evidencia de enfermedad a más de 5 años en el 24, 24응.

Controles por 4 años en el $\quad 9.09$.

Controles por 3 años en el $9.09 \%$

Controles por 2 años en el $24.24 \%$

Controles por 1 año en el $15.15 \%$

Sin dato en el H.C. en el $21.21 \%$

Las recidivas locales se presentaron en el $15.15 \%$

Son factores de importancia pronóstica:

1. Las metastasis linfáticas regionales.

2. El tamaño de la lesión.

3. El sitio de la lesión.

4. El tipo Histológico de la lesión.

Las metastasis regionales reducen la sobrevida del $85 \%$ cuando están ausentes a menos del 50 \% cuando hay un ganglio positivo y a menos del $25 \%$ cuando son varios los ganglios positivos.

TAMAÑo

DE LA LESION

$<1 \mathrm{~cm}$

$>1<2$

$>2<3$

$>3 \mathrm{~cm}$
\%DE

METASTASIS

61.7 
Las lesiones mayores de $3 \mathrm{cms}$ de diámetro tienen un número significativamente mayor de metastasis que las lesiones de menor tamaño.

El grado de diferenciación celular es muy importante, hay correlación entre

\section{BIBLIOGRAFIA}

KRUPP PH. J. Tulane University School of Medicine. Comunicación personal. 1982.

BARCLAY DL, COLLINS CG. HANSEN, L.H. Patient Problem With Cancer of Vulva Am. J. Obstet. Gynecol 84; 1135, 1962.

DISAIA, PH. J. and CREASMAN W.T. Invasive Cancer of the Vulva. Clinical Gynecologic Oncology. The C.V. Mosby Company. 1981. pag. 185 .

JANOVSKI, N.A. DOUGLAS CH, P. Diseases of the Vulva. Harper and Row, Publishers 1972 .

KISTNER, R.W. Tratado de Ginecología. Barcelona Ediciones Toray, 1974.

BREEN J.L. et al. Basal Cell Carcinoma of the Vulva. Am. J. of Obstet and Gynecol Aug. 1975.

CRUM CHIRISTOPHER P. et al. Intraepitelial squamous lesions of the Vulva: Biologic Histologic criteria for the Distinction of los diferentes grados de diferenciación celular con el pronóstico.

En relación al sitio de localización es importante anotar que las lesiones centrales del clítoris son de peor pronóstico que las labiales o laterales.

condylomas From Vulvar intraepithelial Neoplasia. Am J. Obstet. Gynecol. Volume 144: 77 number 1, Septem ber 1, 1982.

IVERSEN TORBJORN. Treatment of inoperable Vulvar Carcinoma. Acta, Obstet, Gynecol Scand 61: 195-197, 1982.

KRUPP_PHILIP (Facog). Therapy of Advanced Epidermoid Carcinoma of Vulva.Obstetrics Gynecology. Vol. 46. No. 4 October 1975.

ORDOÑEZ NELSON et al. Mixed Tumor of the Vulva. A Report of Two Cases Probably Arising in Bartholin's Gland. Cancer, 48: 181-186, 1981.

ABITBOL M. MAURICE. Carcinoma of the Vulva: improvements in the Surgical Approach. Am. J. Obstet Gynecol. Volume 117. Number 4. October 15, 1973.

ULBRIGHT, THOMAS $M$. et al. Bowenoid Displasia of the Vulva. Cancer Vol. 50 2981-2919. December 15, 1982. 